\title{
COMPASS Measurements of Asymmetry Amplitudes in the Drell-Yan Process Observed from Scattering Pions off a Transversely Polarized Proton Target
}

\section{Robert Heitz*}

University of Illinois

E-mail: rsheitz2eilinois.edu

\section{On behalf of the COMPASS Collaboration}

In 2015 the COMPASS collaboration at CERN studied the Drell-Yan process with a $190 \mathrm{GeV} / \mathrm{c} \pi^{-}$beam on a transversely polarized $\mathrm{NH}_{3}$ target. From single-spin asymmetries COMPASS was able to determine amplitudes related to the proton Sivers, transversity and prezelosity transverse momentum dependent (TMD) distributions. The most notable of these TMDs is the semi-universal Sivers function which is theorized to change sign between the Drell-Yan and semi-inclusive deep inelastic scattering (SIDIS) processes. COMPASS has previously measured a non-zero Sivers amplitude from SIDIS and therefore it offers a unique opportunity, with a similar experimental setup and kinematic domain, to definitely conclude on the sign change between the Drell-Yan and SIDIS processes. The analysis and results of the 2015 Drell-Yan COMPASS data are presented.

The European Physical Society Conference on High Energy Physics

\section{5-12 July}

Venice, Italy

\footnotetext{
*Speaker.
} 


\section{Introduction}

The most general way to describe nucleon structure is by Wigner distributions which give all the parameters of a nucleon in a 5 dimensional space describing how partons are bound to make a nucleon [1]. The problem as of now, however, is that it is not possible to access these distributions through experiments. On the other hand, integration over certain parameters in a Wigner distribution gives access to Generalized Parton Distributions (GPD) or Transverse Momentum Dependent (TMD) Parton Distribution Fuctions (PDF). Both GPDs and TMDs are measurable through experiments and the COMPASS collaboration has collected data to measure both of these distributions with lepton beams. Moreover in the summer of 2015 COMPASS collected data to study TMDs and their effects with a hadron beam through the Drell-Yan reaction.

Transverse momentum distributions describe the correlations between the transverse spin of the parent hadron, the spin of the parton and the transverse momentum of a parton. Before the TMD framework, perturbative Quantum Chromodynamics (pQCD) predicted smaller transverse momentum effects than what was observed in experiments [2]. The TMD description improved on pQCD by including soft gluon exchange to account for the transverse momentum of partons which allowed for a better overall description of nucleon structure.

At the leading twist there are eight TMDs that describe a nucleon with two noteworthy TMDs being the Sivers function [3] and the Boer-Mulders [4] function. The Sivers function gives a correlation between the transverse momentum of a parton and the transverse spin of the parent hadron and the Boer-Mulders function gives a correlation between transverse momentum of the parton and the transverse spin of the same parton. The Sivers and the Boer-Mulders distributions are interesting because they are theorized to change sign between the semi-inclusive deep inelastic scattering (SIDIS) process and the Drell-Yan process. This semi-universality arises because the Sivers and Boer-Mulders functions are time reversal odd and in the Drell-Yan process there are only initial state gluons while in SIDIS there are only final state gluons [5]. Comparing the Sivers function or the Boer-Mulders function between the SIDIS and the Drell-Yan processes is therefore a fundamental test of the underlying TMD and QCD theory.

COMPASS has previously studied SIDIS data from a transversely polarized target and measured a non-zero amplitude related to the Sivers function from the SIDIS process [6]. This means COMPASS has the best ability to compare SIDIS and Drell-Yan processes with a similar experimental setup and minimum systematic uncertainties to verify or disprove the semi-universality of the Sivers function.

\section{2015 COMPASS Data Taking}

The COMPASS spectrometer is a two-stage spectrometer where the first stage, the Large Angle Spectrometer (LAS), detects particle tracks from the beam with a polar scattering angle between $26 \mathrm{mrad}$ and $160 \mathrm{mrad}$ and the second stage, the Small Angle Spectrometer (SAS), detects tracks up to a polar scattering angle of $45 \mathrm{mrad}$ [7]. In 2015 COMPASS collided a $190 \mathrm{GeV} / \mathrm{c} \pi^{-}$beam on a transversely polarized ammonia, $\left(\mathrm{NH}_{3}\right)$, target. The average polarization throughout the data taking was 0.73 and a $0.6 \mathrm{~T}$ dipole magnet was used to maintain target polarization. The target was separated into two $55 \mathrm{~cm}$ cells of radius $2 \mathrm{~cm}$ which were in turn separated by $20 \mathrm{~cm}$ and oppositely 
polarized, as shown in figure 1. In 2015, COMPASS took nine data periods where each data period lasted two weeks and the spin orientation of the targets was reversed after the first week of every period to reduce systematic effects arising from different geometric acceptances and luminosities of up and downstream target cells [8].

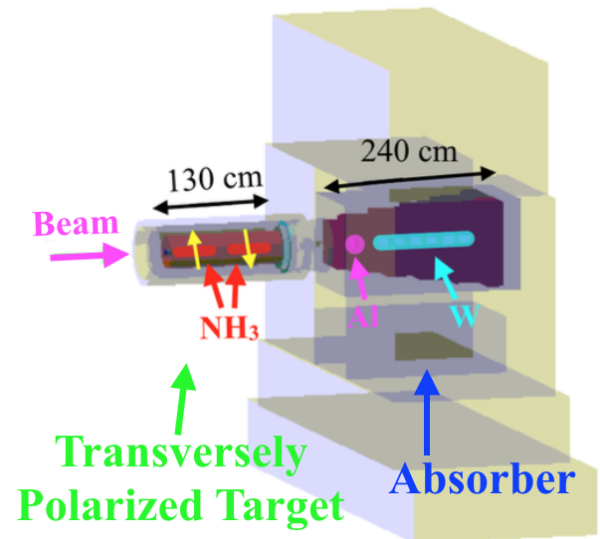

Figure 1: The 2015 hadron absorber ensured the final state only included muons.

The setup also included a hadron absorber placed just downstream of the target cells to drastically reduce the amount of hadrons and electrons detected in the spectrometer and therefore ensure a clean dimuon sample. This absorber was made of alumina $\left(\mathrm{Al}_{2} \mathrm{O}_{3}\right)$ and inside the absorber there was an aluminum plug followed by a tungsten plug as shown in figure 1. The aluminum and tungsten plugs served the double purposes of absorbers and also as unpolarized nuclear targets.

\section{The Drell-Yan Process}

The Drell-Yan process was first theoretically described in 1970 to explain the annihilation of a quark and an anti-quark in the intial state and a resulting final state lepton pair [9]. One of the defining characteristics of the Drell-Yan process is a steep falloff of the cross-section as a function of the final state dilepton invariant mass. Above $4 \mathrm{GeV} / \mathrm{c}^{2}$ the cross-section is of the order of nanobarns. This makes the Drell-Yan process experimentally challenging because high luminosity is needed to achieve sufficient statistics while the background should still be well suppressed.

One of the most interesting features of the Drell-Yan process is its ability to access TMDs experimentally. The leading-order Drell-Yan cross section for an unpolarized beam on a transversely polarized target is shown as a proportionality as follows:

$$
\begin{aligned}
\mathrm{d} \sigma^{\text {Drell-Yan }} \propto & 1+A_{U}^{\cos (2 \phi)} \cos (2 \phi) \\
& +\left|\mathrm{S}_{\mathrm{T}}\right|\left\{A_{T}^{\sin \left(\phi_{S}\right)} \sin \left(\phi_{S}\right)+A_{T}^{\sin \left(2 \phi+\phi_{S}\right)} \sin \left(2 \phi+\phi_{S}\right)+A_{T}^{\sin \left(2 \phi-\phi_{S}\right)} \sin \left(2 \phi-\phi_{S}\right)\right\},
\end{aligned}
$$

where $\left|S_{T}\right|$ is the transverse polarization percentage and the azimuthal amplitudes shown in color are proportional to a convolution of a pion TMD and a proton TMD as follows: $A_{U}^{\cos (2 \phi)} \propto$ Boer-Mulders pion $\otimes$ Boer-Mulders proton, $A_{T}^{\sin \left(\phi_{S}\right)} \propto$ PDF pion $\otimes$ Sivers proton, $A_{T}^{\sin \left(2 \phi+\phi_{S}\right)} \propto$ Boer-Mulders pion $\otimes$ prezelosity and $A_{T}^{\sin \left(2 \phi-\phi_{S}\right)} \propto$ Boer-Mulder pion $\otimes$ transversity [10]. Studying 
Drell-Yan data with the 2015 COMPASS setup of a transversely polarized target and an unpolarized pion beam will allow the extraction of amplitudes related to the proton Sivers, Boer-Mulders, prezelosity and transversity TMD distributions.

\section{COMPASS Drell-Yan Analysis}

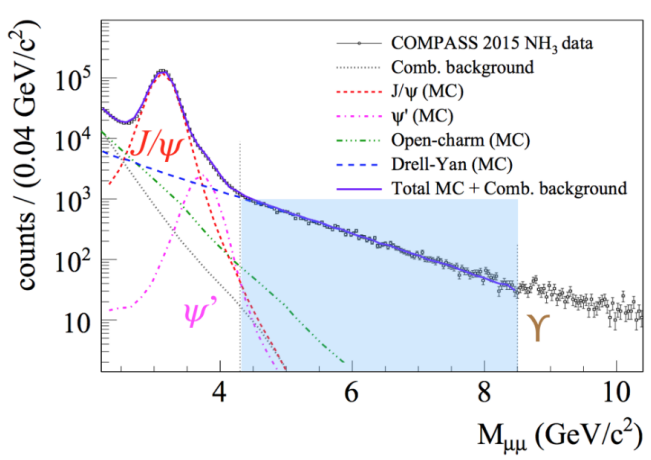

Figure 2: The dimuon invariant mass distribution where the analysis region is highlighted in blue.

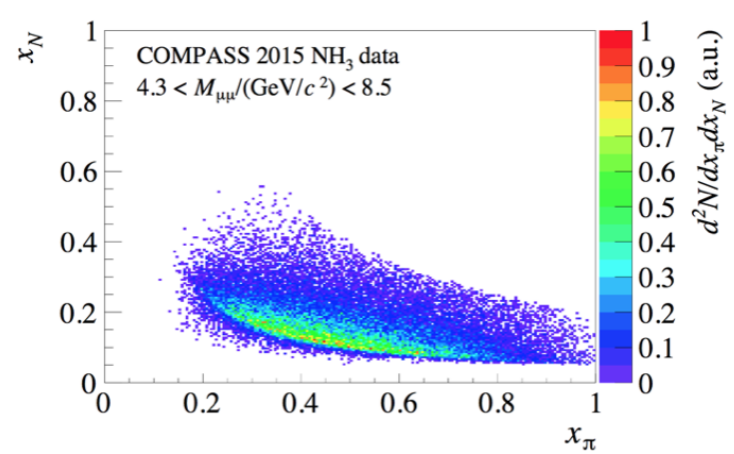

Figure 3: x-Bjorken pion versus x-Bjorken nucleon distribution.

The 2015 COMPASS Drell-Yan data taking set consisted of about $35 \times 10^{3}$ dimuon pairs. The COMPASS collaboration performed their analysis on dimuon events in the high mass range between 4.3 and $8.5 \mathrm{GeV} / \mathrm{c}^{2}$. As can be seen in figure 2 the low invariant mass background contributions from combinatorial background, open charm, $\mathrm{J} / \Psi$ and $\Psi$ ' are well suppressed in this mass range and as well this mass range is safely below the $\Upsilon$ invariant mass. The background in this analysis was estimated to be less than $4 \%$. As can be seen in figure 3 this kinematic region probes the valence region of the target quark with an average $x$-Bjorken above 0.1. In addition the probed Drell-Yan phase space has kinematic overlap with the SIDIS phase space of this experiment and therefore the comparison between these two processes does not need to take evolution effects into account.

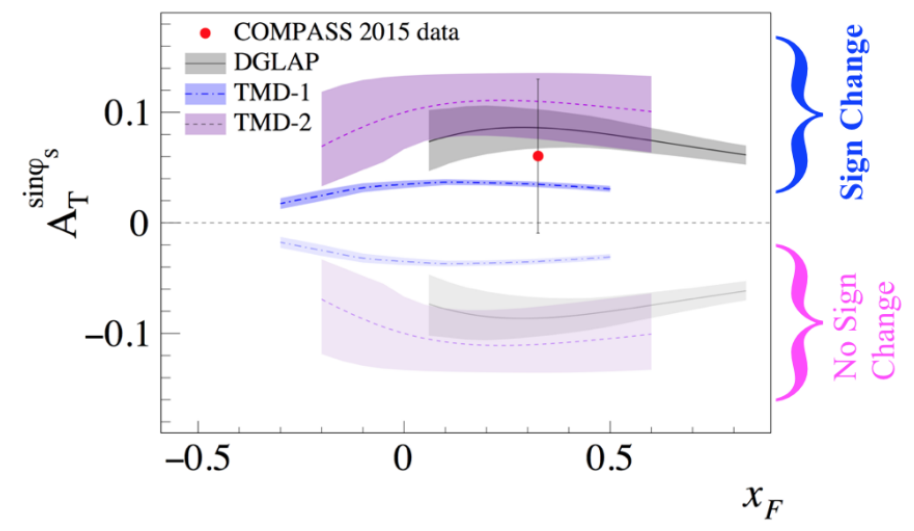

Figure 4: The Sivers amplitude measured at COMPASS shown with the theory predictions for sign change and no sign change. 
Figure 4 shows the amplitude of the Sivers function with statistical and systematic errors along with theory curves determined from the SIDIS process both for a sign change and for no sign change. As figure 4 shows, COMPASS measured the sign and amplitude of the Sivers amplitude to be consistent with the theorized sign flip. Furthermore while the Sivers amplitude was measured to be only 1 sigma away from zero, it was found to be 2 sigma away from being consistent with no sign flip [8].

\section{Conclusion}

From the 2015 transversely polarized Drell-Yan data COMPASS determined the first spindependent azimuthal asymmetries. In addition the COMPASS collaboration has performed the first measurements of transversely polarized SIDIS and Drell-Yan in a similar kinematic region and found the Sivers amplitude determined from each of these processes to be consistent with the theorized sign flip. This is not the end of this study at COMPASS however. COMPASS will perform another spin-dependent Drell-Yan run in 2018 to further strengthen the significance of the results on the spin-dependent azimuthal asymmetries.

\section{References}

[1] Wigner E. Phys. Rev. 40:749 (1932)

[2] M. Grosse Perdekamp and F. Yuan, Ann. Rev. Nucl. Part. Sci. 65, 429 (2015), arXiv:1209.2803 [hep-ph].

[3] D. Sivers, Phys. Rev. D41, 83 (1990).

[4] B. Mulders, Phys. Rev. D57 (1998).

[5] J. Collins, Phys. Lett. B 536 (2002) 43.

[6] C. Adolph et al. (COMPASS Collaboration), Phys. Lett. B770 (2017), arXiv:1609.07374 [hep-ex].

[7] P. Abbon et al. (COMPASS Collaboration), Nucl. Instrum. Meth. A577 (2007) 455-518, arXiv:0703049 [hep-ex].

[8] M. Aghasyan et al. (COMPASS Collaboration), Phys. Rev. Lett. 119 (2017) 112002.

[9] I. Kenyon, Reports on Progress in Physics 45 (1982).

[10] S. Arnold and A. Metz, Phys. Rev. D79 (2009), arXiv:0809.2262v2 [hep-ex]. 